Keywords: $\mathrm{TiO} 2$ nanocontainers, $\mathrm{TiO} 2$ nanospheres, photoreduction of $\mathrm{CO} 2$, photoelectrochemical water splitting

\section{MS20-05 The influence of mobile monovalent ions on structures and properties of multinary thermoelectric tellurides}

Oliver Oeckler ${ }^{1}$, Paul Heitjans ${ }^{2}$, Stefan Schwarzmüller ${ }^{1}$, Andre Düvel $^{2}$, Leonie Schmohl ${ }^{1}$, Matthias Jakob ${ }^{1}$, Alexander Kuhn ${ }^{1}$, Markus Nentwig ${ }^{1}$, Thorsten Schröder ${ }^{3}$

1. Universität Leipzig, Faculty of Chemistry and Mineralogy, IMKM, Scharnhorststr. 20, D-04275 Leipzig, Germany

2. Leibniz Universität Hannover, Institute of Physical Chemistry and Electrochemistry, Callinstr. 3-3a, D-30167 Hannover, Germany

3. Technische Universität München, Physik Department / Heinz Maier-Leibnitz Zentrum, Lichtenbergstr. 1, D-85748 Garching, Germany

email: oliver.oeckler@gmx.de

Crystal structures and thermoelectric properties in the system (GeTe) Sb $_{2} \mathrm{Te}_{3}$ (GST materials) are dominated by high concentrations ${ }^{3}$ of vacancies associated with diffusion-controlled phase transitions.[1] Partially replacing Ge by twice the amount of monovalent cations $\mathrm{M}^{+}$reduces the number of vacancies and yields stable compounds without reconstructive phase transitions. In the case of $\mathrm{Li}$, the lattice thermal conductivity is as low as that of compounds with high vacancy concentrations, indicating that $\mathrm{Li}$ acts as a "pseudo vacancy".[2] Such materials reach thermoelectric figures of merit ZT up to ca. 1. Varying the ratio of $\mathrm{M}^{+}, \mathrm{Ge}^{2+}$ and $\mathrm{Sb}^{3+}$ enables the adjustment of the vacancy concentration and consequently the $\mathrm{Li}$ mobility. Thus, $\mathrm{LiGe}_{3.5} \mathrm{Sb}_{2} \mathrm{Te}_{7}$ or $\mathrm{LiGe}_{115} \mathrm{Sb}_{2} \mathrm{Te}_{15}$ exhibit lower thermal conductivities (0.4 and $1.6 \mathrm{Wm}^{-1} \mathrm{~K}^{-1}$, respectively) than the vacancy-free variants $\mathrm{Li}_{2} \mathrm{Ge}_{3} \mathrm{Sb}_{2} \mathrm{Te}_{7}$ and $\mathrm{Li}_{2} \mathrm{Ge}_{11} \mathrm{Sb}_{2} \mathrm{Te}_{15}$ (1.3 and 2.5 $\left.\mathrm{Wm}^{-1} \mathrm{~K}^{-1}\right)$. As corroborated by superionic $\mathrm{Li}$-ion conductivity in $\mathrm{Li}_{2} \mathrm{Te}$, the mobility of $\mathrm{Li}$ may lead to PLEC (phonon liquid/electron crystal) behavior at elevated temperature. ${ }^{7} \mathrm{Li}$ solid-state NMR spectra clearly indicate Li mobility. Motional narrowing of the NMR signal sets in at temperatures slightly above room temperature. Compounds with vacancies exhibit higher $\mathrm{Li}$ mobilities, correlation times are in the order of magnitude of $10^{-3} \mathrm{~s}$ at ca. $300 \mathrm{~K}$ and less at higher temperatures.

Using $\mathrm{Na}$ instead of $\mathrm{Li}$ is also possible whereas samples with $\mathrm{Cu}$ contain precipitates of copper tellurides. The interplay of doping GST and nanoscale heterostructures leads to ZT values of 1.5 and higher.

Since SnSe emerged as a promising and cheap thermoelectric material,[3] related substitution strategies in the system $\mathrm{Sn} / \mathrm{Sb} / \mathrm{Se}$ seem intriguing. $\mathrm{SnSb}_{2} \mathrm{Se}_{4}$ with a chain-like "sulfosalt" structure [4] is an n-type semiconductor with a high Seebeck coefficient; however, the ZT value is limited by the low electrical conductivity, similar to that of comparable sulfosalts. Formally adding $\mathrm{Na}_{2} \mathrm{Se}$ leads to p-type $\mathrm{Na}_{2} \mathrm{SnSb}_{2} \mathrm{Se}_{5}$ which crystallizes in a NaCl-type structure and 1 surprisingly stable against air and moisture.

[1] T. Rosenthal, M. N. Schneider, C. Stiewe, M. Döblinger, O. Oeckler, Chem. Mater. 2011, 23, 4349.

[2] T. Schröder, S. Schwarzmüller, C. Stiewe, J. de Boor, M. Hölzel, O. Oeckler, Inorg. Chem. 2013, 52, 11288.

[3] H. Zhang, D. V. Talapin, Angew. Chem. Int. Ed. 2014, 53, 9126 . 
[4] P. P. K. Smith, J. B. Parise, Acta Crystallogr. Sect. $B$ 1985, 41,84 .

Keywords: thermoelectrics, tellurides, defects, 7Li NMR
MS21 Structural disorder and

materials' properties at ambient

and non-ambient conditions
Chairs: Dmitry Chernyshov, Vaughan Gavin

1. Laboratory for Neutron Scattering and Imaging, Paul Scherrer Institute, Villigen, Switzerland

2. Department of Quantum Matter Physics, University of Geneva, Switzerland

3. European Synchrotron Radiation Facility, Grenoble, France

4. Swiss-Norwegian Beamlines at European Synchrotron Radiation Facility, Grenoble, France

email: bjorn.wehinger@unige.ch

Diffuse scattering from thermally populated phonons contains important details on the lattice dynamics. The intensity distribution of thermal diffuse scattering across reciprocal space allows for the identification and localization of phonon anomalies and naturally encodes the phonon eigenvectors [1]. In this presentation, the audience will be introduced to the theoretical background, recent developments on model calculations and the use of pertinent software for computing the intensity distribution in 3D reciprocal space [2]. Important aspects of the experimental implementation and data treatment are discussed and the methodology is illustrated by a representative set of recent examples [3-5]. I will show how distinct features in the lattice dynamics leave their footprint in the intensity distribution, such as soft and low energy phonon modes (see Figure 1), similarities in the electronic potential and symmetry relations upon phase transitions. Finally, the possibility for extracting the full elasticity tensor from thermal diffuse scattering is discussed and an outlook to application at high pressures is presented.

[1] A. Bosak et al 2015 In-between Bragg reflections: thermal diffuse scattering and vibrational spectroscopy with x-rays J. Phys. D: Appl. Phys. 48504003

[2] B. Wehinger and A. Mirone 2013-2016, ab2tds, https://forge.epn-campus.eu/html/ab2tds/Introduction.html

[3] B. Wehinger, A. Bosak, and P. T. Jochym 2016 Soft phonon modes in rutile $\mathrm{TiO}_{2}$ Phys. Rev. B 93, 014303

[4] B. Wehinger et al 2015 Lattice dynamics of $\alpha$-cristobalite and the Boson peak in silica glass $J$. Phys.: Condens. Matter 27305401

[5] B. Wehinger et al 2014 Diffuse scattering in metallic tin polymorphs J. Phys.: Condens. Matter 26 115401 